\title{
Cytokines and Vascular Mediators Fingerprints Based on Interaction Networks in Kidney Transplanted and Non-Transplanted Moderately III COVID-19 Patients
}

\author{
Milena Karina Coló Brunialti \\ Universidade Federal de Sao Paulo \\ Giuseppe Gianini Figueirêdo Leite \\ Universidade Federal de Sao Paulo \\ Orlei Ribeiro Araújo \\ GRAACC, Universidade Federal de Sao Paulo

\section{Gabriela Strafolino Ebumeo} \\ Universidade Federal de Sao Paulo \\ Paula M Peçanha-Pietrobom \\ Universidade Federal de Sao Paulo \\ Paulo Roberto Abrão Ferreira \\ Universidade Federal de Sao Paulo \\ Nancy Junqueira Bellei \\ Universidade Federal de Sao Paulo \\ Jaquelina Sonoe Ota Arakaki \\ Universidade Federal de São Paulo \\ Lúcio R. Requião-Moura \\ Universidade Federal de São Paulo \\ Reinaldo Salomao ( $\sim$ rsalomao@unifesp.br) \\ Universidade Federal de Sao Paulo
}

Research Article

Keywords:

Posted Date: January 17th, 2022

DOI: https://doi.org/10.21203/rs.3.rs-1184252/v1

License: () This work is licensed under a Creative Commons Attribution 4.0 International License. Read Full License 


\section{Abstract}

This prospective study enrolled 68 hospitalized COVID-19 patients (including 20 kidney transplant recipients, KTRs) and 29 controls, and a panel comprising 23 circulating cytokines and vascular mediators was run at different time points. The associations between in-hospital mortality and mediators were determined using logistic regression and correlation-based network (CBN) analysis. In total, 17.6\% of patients died, and the in-hospital mortality was associated with early and over-time levels of several mediators. Death was predicted by SVCAM, IL-9, and KTR in the multivariable model (AUC-ROC=0.94). Overall, circulating mediator levels were similar in the non-KTR and KTR COVID-19 patients. Compared to their respective controls, the number of interactions in the networks increased more in non-KTRs (14-55) than in KTRs (16-26), with a higher positive/negative edges (pe/ne) ratio in non-KTRs than in KTRs patients. The correlation network was also more connected in survivors than in non-survivors. Changing the pe/ne ratio from the first to the third day of hospitalization seems to be associated with survival: from 12 to 21.5 among survivors and from 4.0 to 2.37 among nonsurvivors. In conclusion, in-hospital mortality was associated with increased levels of cytokines and vascular mediators, but survivors and non-KTR patients showed more densely connected CBNs.

\section{Introduction}

The interaction of severe acute respiratory syndrome coronavirus 2 (SARS-CoV-2) with target cells, the increased circulating cytokine levels, and the imbalance between pro and anti-inflammatory mediators seem to be a cornerstone of coronavirus 2019 (COVID-19) severity ${ }^{1-3}$; Therefore, cytokines storm have been considered pivotal events in COVID-19's disease pathophysiology and as possible targets for therapy ever since the pandemic was declared ${ }^{4,5}$. Beyond cytokine storms, microangiopathy, endothelial dysfunction, and widespread thrombosis are also observed in patients who deteriorate during the course of the disease ${ }^{6-8}$. Nevertheless, similarities and differences between sepsis and COVID-19 regarding cytokine-induced disturbances in coagulation ${ }^{9-11}$ and thrombosis mechanisms have been demonstrated ${ }^{12,13}$, few reports have addressed changes and correlations in cytokines, endothelial, and coagulation mediators in COVID-19.

Furthermore, since the first reports, clinical presentations and outcomes in some clusters of patients, such as kidney transplant recipients (KTRs), have awakened particular concerns due to their unavoidable lifelong immunosuppression maintenance and cumulative number of comorbidities ${ }^{14,15}$. High vulnerability has been demonstrated in several cohorts, resulting in high hospitalization rates and impressive case fatality rates ${ }^{16,17}$. Despite these impacting results, the presence of the kidney graft per se and the effect of chronic use of immunosuppressive agents in the imbalance between cytokine storm, microangiopathy, thrombosis, and, consequently, in the inflammatory response and endothelial dysfunction, is not known and has not been previously investigated.

We prospectively followed a cohort of COVID-19 patients, focusing on those affected by moderate disease, including KTRs, admitted to a tertiary hospital in Brazil. The clinical and epidemiological characteristics and routine laboratory changes have been previously published ${ }^{18}$. Herein, we evaluated the plasma levels of cytokines and vascular mediators assessed at hospital admission and at different time points in the course of the disease, aiming to describe their signature in those patients, trace possible differences according to kidney transplant status, and seek early predictors of severity and in-hospital mortality.

\section{Results}

\section{Baseline characteristics and outcomes}

Patients were $59.2(50.1-66.8)$ years, and most of them were men (61.8\%). The healthy control (HC) individuals ( $\mathrm{n}=19)$ were $58(50-64.5)$ years old and predominantly male (63\%), whereas the KTR controls (KTRcs) were 58.1 (50.5-59.1) years old and 60\% were male. The demographic data and clinical details of this cohort have already been published ${ }^{18}$.

Deterioration to critical disease was observed in 35.3\% ( $n=24)$, and 22.1\% ( $n=15)$ required MV. In-hospital mortality was 17.6\% ( $n=12,8$ KTRs). For survivors, the median length of hospital stay was 9 (5-15) days.

\section{Cytokines and vascular mediators}

Higher levels of cytokines and vascular mediators were observed in COVID-19 patients than in controls on the first day of hospitalization. The levels were similar between non-KTR and KTR patients, except for higher mean IL-9 values and lower mean P-Sel values in KTR patients. The main results are depicted in Figure 1 and Figure S1 in Supplementary Material 1.

\section{Predictors of outcomes}

In the first step, the predictive capacity of the mediators on the first and third days after hospital admission for KTRs in terms of the outcomes were evaluated using bivariate and multivariate logistic regression models. The significant bivariate models using the mediators assessed on the first day are shown in Table 1, and an extra model for in-hospital death was fitted considering the mediators on the third day. The Hosmer and Lemeshow goodness of fit $P$-value was 0.82 , and a logit equation based on that was $Y=-7.47+($ sVCAM ng $/ \mathrm{mL} \times 0.003)+(\mathrm{IL}-9 \mathrm{pg} / \mathrm{mL} \times 0.47)+(\mathrm{kidney}$ transplant $[0=\mathrm{no}$ or $1=y e s] \times 3.25)$. The model achieved an AUC-ROC of $0.94(95 \% \mathrm{Cl}: 0.87-1, p<0.05)$ in predicting in-hospital mortality. 
Table 1

Logistic regression models for in-hospital mortality, mechanical ventilation requirement and intensive care unit admission.

\section{Bivariate logistic regression models with first day mediators levels}

Outcome: in-hospital mortality

\begin{tabular}{lllll} 
Predictor & Estimate & OR & $95 \% \mathrm{Cl}$ & $P$ \\
\hline GDF-15 & 0.16 & 1.17 & $1.01-1.34$ & 0.026 \\
\hline sICAM-1 & 0.008 & 1.01 & $1-1.015$ & 0.007 \\
\hline NGAL & 0.008 & 1.01 & $1-1.015$ & 0.036 \\
sVCAM & 0.0004 & 1.0004 & $1.0001-1.0008$ & 0.016 \\
\hline Kidney transplant & 1.95 & 7.16 & $1.79-27.3$ & 0.005
\end{tabular}

Outcome: mechanical ventilation requirement

\begin{tabular}{|c|c|c|c|c|}
\hline Predictor & Estimate & OR & $95 \% \mathrm{Cl}$ & $P$ \\
\hline IL-6 & 0.004 & 1.004 & $1.001-1.008$ & 0.009 \\
\hline sICAM-1 & 0.006 & 1.006 & $1-1.01$ & 0.03 \\
\hline NGAL & 0.008 & 1.008 & $1-1.01$ & 0.03 \\
\hline Kidney transplant & 1.5 & 4.6 & $1.32-15.7$ & 0.016 \\
\hline \multicolumn{5}{|c|}{ Outcome: critical illness } \\
\hline Predictor & Estimate & OR & $95 \% \mathrm{Cl}$ & $P$ \\
\hline IL-6 & 0.004 & 1.004 & $1.001-1.008$ & 0.009 \\
\hline \multicolumn{5}{|c|}{ Multivariate logistic regression models with third day mediators' levels } \\
\hline \multicolumn{5}{|c|}{ Outcome: in-hospital mortality } \\
\hline Predictor & Estimate & OR & $95 \% \mathrm{Cl}$ & $P$ \\
\hline IL-9 & 0.47 & 1.6 & $1.06-2.42$ & 0.025 \\
\hline sVCAM-1 & 0.003 & 1.003 & $1.001-1.005$ & 0.01 \\
\hline Kidney transplant & 3.3 & 26.9 & $2.2-329.6$ & 0.01 \\
\hline Intercept & -7.54 & - & - & 0.01 \\
\hline
\end{tabular}

Abbreviations: GDF-15, growth/differentiation factor 15; Cl, confidence interval; IL-6, interleukin 6; NGAL, neutrophil gelatinase-associated lipocalin; OR, odds ratio; sICAM-1, soluble intercellular adhesion molecule-1; sVCAM-1, soluble circulating vascular cell adhesion molecule-1.

In the second step, the repeated mediator measures as the within-subjects factors obtained using the generalized estimating equations were fitted with severity (progressing or not to critical illness), MV requirement, and in-hospital mortality as binary responses. First, as shown in Table 2, lower means of ADAMTS-13, P-Sel, IL-1 $\beta$, and IL-2, as well as higher means of GDF-15, MYO, sICAM-1, MPO, NGAL, sVCAM-1, IL-6, IL-8, and IL-18 were predictors of severity. Subsequently, lower means of ADAMTS-13 and P-Sel and higher means of GDF-15, sICAM-1, MPO, NGAL, sVCAM-1, IL-6, IL-8, and IL-18 were predictors of MV requirement. Lastly, higher means of GDF-15, MYO, sICAM-1, MPO, NGAL, sVCAM-1, IL-6, IL-8, IL-9, IL-18, and lower means of IL-2 and IL-4 predicted in-hospital death. 
Table 2

The variations in the levels of mediators over time points and clinical outcomes. Repeated measures were used as the within-subjects factor in the generalized estimating equation models for death. Only the significant results of the estimated effect and different estimated means are shown. Fields

\begin{tabular}{|c|c|c|c|c|c|c|c|c|c|c|c|c|}
\hline Mediators & Severe & Estimate & Mean & $P$ & MV & Estimate & Mean & $P$ & Death & Estimate & Mean & $P$ \\
\hline \multirow{2}{*}{$\begin{array}{l}\text { ADAMTS- } \\
13\end{array}$} & No & 0.2 & 676.7 & 0.003 & No & 0.3 & 669.8 & $<0.001$ & & & & \\
\hline & Yes & & 562.9 & & Yes & & 513.8 & & & & & \\
\hline \multirow[t]{2}{*}{ GDF } & No & -1.2 & 2.0 & $<0.001$ & No & -1.2 & 2.2 & $<0.001$ & No & -1.3 & 2.3 & $<0.001$ \\
\hline & Yes & & 6.0 & & Yes & & 7.4 & & Yes & & 9.1 & \\
\hline \multirow[t]{2}{*}{ Myo } & No & -2.6 & 48.6 & $<0.001$ & & & & & No & -2.2 & 126.0 & 0.005 \\
\hline & Yes & & 669.8 & & & & & & Yes & & 1169.5 & \\
\hline \multirow[t]{2}{*}{ sICAM-1 } & No & -0.8 & 79.6 & $<0.001$ & No & -146.4 & 83.8 & 0.004 & No & -0.5 & 104.2 & 0.016 \\
\hline & Yes & & 181.7 & & Yes & & 230.3 & & Yes & & 176.2 & \\
\hline \multirow[t]{2}{*}{ MPO } & No & -0.3 & 61.9 & 0.008 & No & -21.8 & 63.9 & 0.018 & No & -0.5 & 62.5 & $<0.001$ \\
\hline & Yes & & 81.0 & & Yes & & 85.7 & & Yes & & 103.5 & \\
\hline \multirow[t]{2}{*}{ P-Sel } & No & 0.4 & 148.5 & 0.008 & No & 37.1 & 139.2 & 0.047 & & & & \\
\hline & Yes & & 101.7 & & Yes & & 102.1 & & & & & \\
\hline \multirow[t]{2}{*}{ NGAL } & No & -0.7 & 58.1 & $<0.001$ & No & -80.7 & 65.6 & $<0.001$ & No & -8 & 71.7 & $<0.001$ \\
\hline & Yes & & 120.4 & & Yes & & 146.3 & & Yes & & 153.4 & \\
\hline \multirow[t]{2}{*}{ sVCAM-1 } & No & -0.4 & 1300.3 & 0.004 & No & -1023.9 & 1302.4 & $<0.001$ & No & -0.7 & 1318.3 & $<0.001$ \\
\hline & Yes & & 1940.5 & & Yes & & 2326.4 & & Yes & & 2725.7 & \\
\hline \multirow[t]{2}{*}{ IL-1 $\beta$} & No & 2.1 & 27.1 & 0.032 & & & & & & & & \\
\hline & Yes & & 3.5 & & & & & & & & & \\
\hline \multirow[t]{2}{*}{ IL-2 } & No & 1.9 & 39.2 & 0.023 & & & & & No & 1.9 & 28.7 & 0.026 \\
\hline & Yes & & 5.9 & & & & & & Yes & & 4.3 & \\
\hline \multirow[t]{2}{*}{ IL-4 } & & & & & & & & & No & 1.7 & 28.3 & 0.045 \\
\hline & & & & & & & & & Yes & & 5.1 & \\
\hline \multirow[t]{2}{*}{ IL-6 } & No & -107.1 & 57.5 & 0.034 & No & -186.6 & 55.89 & 0.010 & No & -0.9 & 78.4 & 0.012 \\
\hline & Yes & & 164.7 & & Yes & & 242.47 & & Yes & & 200.9 & \\
\hline \multirow[t]{2}{*}{ IL-8 } & No & -29.7 & 18.1 & 0.002 & No & -42.9 & 19.20 & 0.003 & No & -0.9 & 23.3 & $<0.001$ \\
\hline & Yes & & 47.8 & & Yes & & 62.11 & & Yes & & 56.1 & \\
\hline \multirow[t]{2}{*}{ IL-9 } & & & & & & & & & No & -0.6 & 2.9 & 0.008 \\
\hline & & & & & & & & & Yes & & 5.1 & \\
\hline \multirow[t]{2}{*}{$\mathrm{IL}-18^{\mathrm{a}}$} & No & -0.4 & 656.2 & 0.030 & No & -667.9 & 646.09 & 0.003 & No & -561.7 & 701.0 & 0.027 \\
\hline & Yes & & 952.2 & & Yes & & 1314.03 & & Yes & & 1262.3 & \\
\hline
\end{tabular}

Finally, a model for predicting the severity and probability of death was fitted by considering the differences between KTRs and non-transplant patients. Among non-transplanted patients, lower means of ADAMTS-13 and P-Sel and higher means of GDF-15, MYO, sICAM-1, MPO, NGAL, sVCAM-1, IL-8, and IL-18 predicted severity, while higher means of GDF-15, MPO, sVCAM, and IL-18 predicted death. On the other hand, for KTRs, lower means of ADAMTS13 and higher means of MYO, MPO, NGAL, IL-6, IL-8, and IL-10 predicted severity, whereas higher means of GDF-15, myoglobin, sICAM, MPO, NGAL, sVCAM-1, IL-6, IL-8, IL-10, and IFN-a predicted death. The models are presented in Supplementary Material 2.

Principal component analysis 
In a principal component analysis (PCA), two main factors explained $75 \%$ of the total variance of the cytokines and vascular mediators, with a KaiserMeyer-Olkin factor adequacy of 0.86 for the measures assessed on the first day $(P<0.0001$ in the Bartlett test). For the levels of mediators assessed at all time points (from the 1 st to after discharge), the reduction of two principal components explained $77 \%$ of the total variance (Kaiser-Meyer-Olkin factor adequacy of $0.88 ; P<0.0001)$. IL-18 was not included in this analysis because it was not measured in KTRs. The rotated Varimax solution on the first day and repeated measurements at all time points are shown in Supplementary Material 3.

\section{Network analyses}

To evaluate the relationship between the levels of cytokines and vascular mediators during SARS-CoV-2 infection in KTR and non-KT patients, four correlation-based networks (CBNs) were constructed (Figure 2). The results for the total of interactions and the positive edges to negative edges (pe/ne) ratios were, respectively: 14 and 6 in HCs (Figure 2a), 16 and 15 in KTRcs (Figure 2b); 55 and 8.16 for non-KTRs with COVID-19 (Figure 2c); and 26 and 3.3 for KTRs with COVID-19 (Figure 2d). Interestingly, a higher number of interactions among the cytokines and vascular mediators was observed in COVID-19 patients (Figure 2c and 2d) than in the respective controls (Figure 2a and 2b). The number of interactions was even more prominent in nonKTRs than in KTRs (Supplementary Material 4).

The triggering of cytokines and vascular mediators during COVID-19 might result in a complex interaction network, which may be related to patient outcomes. Therefore, the patients were divided into two groups (survivor and non-survivor) and two different time points (first and third days) for the next analysis. The correlation network between cytokines and vascular mediators was more connected in the survivors (Figure 3a and 3c) than in nonsurvivors (Figure $3 \mathrm{~b}$ and $3 \mathrm{~d}$ ). From the first to the third day, the number of interactions decreased from 52 to 45 , while the pe/ne ratio increased from 12 to 21.5 among survivors. On the other hand, during this period, the number of interactions increased from 15 to 27 , while the pe/ne ratio reduced from 4.0 to 2.37 among non-survivors.

Finally, the correlations of the four networks represented in Figure 3 were examined using InteractiVenn, which identified similarities and differences between the correlations. The correlation between P-Sel and G-CSF and between MYO and NGAL was common among the four networks. Notably, the correlation between ADAMTS13 and SAA was positive on the first and third days in survivors and negative on the first day in non-survivors.

\section{Discussion}

Early in the pandemic, the cytokine signature triggered by the SARS-CoV-2 infection was recognized as a cytokine storm, providing opportunities for identifying predictors of clinical deterioration and new targets for clinical interventions. In the present study, we investigated the association of circulating levels of several mediators in moderately ill COVID-19 patients along with the clinical outcomes, including a subset of vulnerable individuals, kidney transplant recipients. Additionally, we investigated the crosstalk between these mediators, seeking patterns of responses based on correlations of cytokine networks.

In the logistic regression models, the levels of GDF-15, sICAM-1, and SVCAM-1 on the first day after hospitalization were associated with death, whereas sICAM-1 was also associated with MV requirement. It is not surprising that adhesion molecules were associated with crude clinical outcomes, because ICAM-1 and VCAM-1 mediate leukocyte and platelet interactions with endothelial cells, eliciting a microvascular response with impaired endotheliumdependent vasorelaxation in arterioles, excess fluid filtration in capillaries, and protein extravasation in venules, resulting in tissue and organ function impairment ${ }^{19,20}$. On the other hand, GDF-15 modulates vascular responses involving the nitric oxide pathway, impairing vascular contractile and relaxing functions and amplifying organ dysfunction ${ }^{21}$. In addition, in a complex and tissue-specific regulation pathway, GDF-15 suppresses antigen presentation by dendritic cells ${ }^{22}$ and is upregulated by IL-1 $\beta$, TNF-a, IL-2, and macrophage colony-stimulating factor- ${ }^{23}$. Interestingly, the level of IL-9 on the third day of hospital admission was independently associated with in-hospital mortality in the multivariate model. IL-9 promotes the expression of VCAM-1 and P-selectin, resulting in platelet aggregation, which plays a crucial role in the development of thrombotic events ${ }^{24}$. Lastly, the levels of IL6 on the first day of hospitalization were related to ICU and MV requirements. Similarly, a meta-analysis that included 10 studies ( $n=1,789$ ) found 3.24fold higher levels of IL-6 in patients who were admitted to the ICU compared to non-severe patients. The role of IL-6, initially considered the core of the cytokine storm, has recently been questioned because the levels of IL-6 in COVID-19 patients are markedly lower than those in patients with acute respiratory distress syndrome due to other etiologies and sepsis ${ }^{25}$.

Beyond a significant association between early circulating levels of some mediators and outcomes, most mediators' changes over time, evaluated by the generalized estimated equation using repeated measures, were associated with clinical deterioration, MV requirement, and death, suggesting that a possible signature is built by the kinetic evaluation of cytokines and vascular mediators, such as MPO, IL-2, and ADAMTS-13. The neutrophil-originated MPO, an enzyme derived from leukocytes that catalyzes the formation of reactive oxidant species, promotes oxidative stress, hypoxia, and DNA damage by hydroxyl radical generation, contributing to COVID-19 severity ${ }^{26}$. On the other hand, low IL-2 levels and decreased expression of IL-2R in peripheral blood mononuclear cells have already been found in critical patients with COVID-19 pneumonia ${ }^{27}$. Finally, we found that non-severe patients and those who did not require MV had higher estimated means of ADAMTS-13, which is essential for regulating the size of von Willebrand factor (vWF) multimers The imbalance between vWF multimers and ADAMTS-13 leads to a prothrombotic state in inflammatory conditions, such as sepsis and disseminated intravascular coagulation, as well as pulmonary microvascular thrombosis and COVID-19 severity ${ }^{28}$.

In the last step, we refined our analysis by seeking the correlation-based networks of cytokines and vascular mediators. It is well known that the levels of several cytokines can predict the clinical course, severity, and prognosis of different inflammatory diseases, mainly in the acute phase of sepsis ${ }^{29-32}$. 
Generally, these associations have been based on mediator levels assessed at determined time points; however, more recently, the visualization of cytokine networks and their association with clinical outcomes have emerged as a milestone in the investigation of some critical processes, better explaining inflammatory and endothelial dysfunction and consequent organ failure ${ }^{33-35}$. In the context of COVID-19, cytokine network analyses have previously demonstrated the association between immune mediators and clinical parameters, such as pneumonia, hypoxia, and ICU admission ${ }^{35}$, similarities between the response to influenza and SARS-CoV-2 infection, and prediction of protein-protein interactions ${ }^{33,35}$. In addition, a higher number of edges in cytokine networks may be associated with severity ${ }^{33}$. In our study, the visualization of cytokine and vascular mediator networks resulted in novel and exciting information, highlighting not only a unique mediator, such as IL-6, but also the patterns of several interactions. For instance, correlations between P-Sel and G-CSF and between MYO and NGAL were observed in all networks. In addition, a positive correlation between ADAMTS13 and SAA was observed among patients who survived, with a negative correlation observed in non-survivors.

Furthermore, we demonstrated that the correlation between cytokines and vascular mediators could represent coordinated responses and that the networks may differ between patients who will survive and those who will not (Figure 3). Despite lower levels of cytokines and vascular mediators, survivors presented more correlations and a lower number of negative correlations from the first to the third day after hospital admission (Figures 3 a and $\mathbf{c}$ versus $\mathbf{3 b}$ and $\mathbf{d}$ ), suggesting that the higher the number of correlations, the more coordinated the response by inflammatory and endothelial mediators. Finally, a similar portrait was observed when the CBNs of transplanted patients were visualized and compared to non-transplanted patients (Figure 2). For these patients, the number of interactions increased from healthy controls to non-KTR COVID-19 patients based on the increase in positive interactions. On the other hand, the number of increased interactions was more modest in KTRs and essentially based on increases in the number of negative interactions, suggesting that transplanted patients presented disorganized patterns of cytokine-vascular mediator interactions. These findings may be related to chronic exposure to different immunosuppressive classes of drugs that drive T-cell activity as well as graft microvasculature.

Our study has some limitations, such as the small number of patients enrolled and the fact that the population data was from a single center. In addition, we did not investigate the influence of viral load or the role of virus variants in the mediators and outcomes. On the other hand, it has some strengths that can be highlighted, such as its prospective design, the narrow scope of clinical presentation at enrollment in the study, and the different clinical outcomes evaluated.

In summary, clinical deterioration and in-hospital death could be predicted by increased circulating levels of several inflammatory and vascular mediators, while survivors and non-KTR patients showed more densely connected CBNs.

\section{Methods}

\section{Design study, population, and setting}

This was a prospective cohort study that enrolled COVID-19 patients admitted to the Hospital São Paulo, Federal University of São Paulo, Brazil, between May and September 2020. The inclusion criteria were age $>18$ years and need for hospital admission to the ward, mostly due to moderate disease, defined according to the World Health Organization (WHO) criteria ${ }^{36}$. All patients had a COVID-19 diagnosis confirmed by polymerase chain reaction (PCR) testing their nasopharyngeal swabs. The study was approved by the research ethics committee at the Federal University of São Paulo (number 4.453.137). Blood samples were collected from patients and healthy volunteers after written informed consent was obtained. Patients' treatment followed the standard hospital protocols. All methods were carried out following specific national legislation, local Institutional Review Board recommendations, and the guidelines of the Declaration of Helsinki.

Sixty-eight patients were enrolled, including 20 that were KTRs. For the control group, 19 healthy volunteers with no symptoms related to COVID-19 and who had negative COVID-19 results from their serological tests and PCR tests were matched based on sex and age (healthy controls). In addition, 10 KTRs with no symptoms related to COVID-19 and no contact with COVID-19 were matched in terms of time since transplantation, immunosuppressive therapy, sex, and age as a further control group.

\section{Outcomes}

The primary outcome was in-hospital mortality, and the intermediate outcomes were clinical progression to critical illness and the requirement for mechanical ventilation (MV).

\section{Blood sampling and plasma detection of circulating mediators}

Blood samples were collected at the following time points: at admission $(n=68)$, on the 3rd $(n=51), 7$ th $(n=37)$, and 21 st $(n=15)$ days, and after hospital discharge in 43 survivors, with a median time of 33 days. Samples were transported at $4^{\circ} \mathrm{C}$ to the research laboratory, processed within two hours, frozen at $-80^{\circ} \mathrm{C}$ after centrifugation, and kept until use. The details of the mediators and methodological specificity are shown in Table 3 . 
Table 3

The panel of cytokines and vascular mediators

\begin{tabular}{|c|c|c|}
\hline $\begin{array}{l}\text { Mediators } \\
\text { groups }\end{array}$ & Mediators & Methods \\
\hline \multirow[t]{14}{*}{ Cytokines } & IL-1 $\beta$ & Cytometric Bead Array (CBA) Flex Set kits \\
\hline & IL-2 & \multirow{12}{*}{$\begin{array}{l}\text { The samples were analyzed in LSRFortessa flow cytometry and FCAP array software } \\
\text { from BD Biosciences, San Jose, CA, USA }\end{array}$} \\
\hline & $\mathrm{IL}-4$ & \\
\hline & IL-6 & \\
\hline & IL-8 & \\
\hline & IL-9 & \\
\hline & IL-10 & \\
\hline & IL-17A & \\
\hline & $\begin{array}{l}\text { Granulocyte colony-stimulating factor (G- } \\
\text { CSF) }\end{array}$ & \\
\hline & $\begin{array}{l}\text { Granulocyte-macrophage colony- } \\
\text { stimulating factor (GM-CSF) }\end{array}$ & \\
\hline & Tumor necrosis factor-alpha (TNF-a) & \\
\hline & Interferon alpha (IFN-a) & \\
\hline & Interferon gamma (IFN-ү) & \\
\hline & $\mathrm{IL}-18^{\mathrm{a}}$ & ELISA (Human IL-18 ELISA Kit, MBL International, Woburn, MA, USA) \\
\hline \multirow{9}{*}{$\begin{array}{l}\text { Vascular } \\
\text { mediators }\end{array}$} & ADAMTS-13 & Cytometry (MAGPIX ${ }^{\circledR}$ Instrument, Luminex Corporation, Austin, TX, USA) \\
\hline & Growth/differentiation factor 15 (GDF-15) & \multirow{8}{*}{$\begin{array}{l}\text { Kit Milliplex Map Human Cardiovascular Disease Magnetic Bead Panel } 2 \text { from } \\
\text { MilliporeSigma (Temecula, CA, USA) }\end{array}$} \\
\hline & Myoglobin (MYO) & \\
\hline & $\begin{array}{l}\text { Soluble intercellular adhesion molecule-1 } \\
\text { (sICAM-1) }\end{array}$ & \\
\hline & Myeloperoxidase (MPO) & \\
\hline & P-selectin (P-Sel) & \\
\hline & $\begin{array}{l}\text { Neutrophil gelatinase-associated lipocalin } \\
\text { (NGAL) }\end{array}$ & \\
\hline & $\begin{array}{l}\text { Soluble circulating vascular cell adhesion } \\
\text { molecule-1 (sVCAM-1) }\end{array}$ & \\
\hline & Serum amyloid $\mathrm{A}$ (SAA) & \\
\hline
\end{tabular}

\section{Statistical analysis}

Differences between means were evaluated using $t$-tests. In addition, bivariate and multivariate logistic regression models for primary and intermediate outcomes were fitted, including the mediators on the first and third days after hospitalization. For the selection of predictive models, the area under the receiver operating characteristic curve (AUC-ROC) and goodness-of-fit by the Hosmer-Lemeshow test were observed. The significance level was set at $P$ $<0.05$.

To estimate the average response of the repeated measures of the cytokines and vascular mediators on the correlated outcomes, generalized estimating equations were run using SPSS software (IBM SPSS, version 20.0, Armonk, NY, USA). The model types for each variable, linear or gamma with the log link function, were defined by minimizing the corrected quasi-likelihood under the independence model criteria. The mediators were also included in a factor analysis model, with principal component analysis, Bartlett's test for sphericity, and the Kaiser-Meyer-Olkin test for sampling adequacy, using the software R (version 4.1.0, The R Foundation for Statistical Computing, Vienna, Austria).

\section{Correlation-based networks analysis}

Correlation networks were based on correlation analysis of cytokines and vascular mediators. Spearman correlation coefficients ( $r$ ) were calculated using the 'Hmisc' R package. Only correlations that achieved a $P<0.05$, and $r>|0.20|$ were considered for the network composition. The list of 
correlations was exported and visualized using Cytoscape (v. 3.8.2) ${ }^{37}$.

\section{Abbreviations}

ADAMTS-13

Disintegrin and metalloproteinase with thrombospondin motifs 13

AUC-ROC

area under the receiver operating characteristic curve

COVID-19

Coronavirus disease 2019

G-CSF

Granulocyte colony-stimulating factor

GDF-15

Growth/differentiation factor 15

GM-CSF

Granulocyte-macrophage colony-stimulating factor

$\mathrm{HC}$

Healthy control

IFN-a

Interferon alpha

IFN-y

Interferon gamma

IL

Interleukin

KTRs

Kidney transplant recipients

KTRcs

Kidney transplant recipients controls

MV

Mechanical ventilation

MPO

Myeloperoxidase

MYO

Myoglobin

NGAL

Neutrophil gelatinase-associated lipocalin

PCA

Principal component analysis

PCR

Polymerase chain reaction

$\mathrm{pe} / \mathrm{ne}$

Positive edges to negative edges ratio

P-Sel

P-selectin

SAA

Serum amyloid $A$

SICAM-1

Soluble intercellular adhesion molecule-1

sVCAM-1

Soluble circulating vascular cell adhesion molecule-1

TNF-a

Tumor necrosis factor-alpha

\section{Declarations}

Acknowledgments: We thank Professor Jose Medina-Pestana from the Nephrology Division at the Federal University of São Paulo and Hospital do Rim for his helpful discussions related to kidney transplantation.

Author contributions: 
MKCB: conception or design of the work; acquisition, analysis, and interpretation of data; drafted the work and substantively revised it; and approved the submitted version. GGFL: analysis, and interpretation of data; drafted the work and substantively revised it; and approved the submitted version. ORA: analysis, and interpretation of data; drafted the work and substantively revised it; and approved the submitted version. GSE: conception or design of the work; acquisition, of data; and approved the submitted version. PRAF: acquisition, of data; and approved the submitted version. NJB: acquisition, and interpretation of data; and approved the submitted version. JSOA: acquisition, and interpretation of data; and approved the submitted version. LRRM: analysis and interpretation of data; drafted the work and substantively revised it; and approved the submitted version. RS: conception or design of the work; acquisition, analysis, and interpretation of data; drafted the work and substantively revised it; and approved the submitted version.

Competing Interests: The authors declare that they have no conflict of interest.

\section{Funding:}

This work was supported by Fundacao de Amparo à Pesquisa do Estado de São Paulo (FAPESP), grant numbers 2017/21052-0 and 2020/05110-2 to RS. GL has a scholarship from FAPESP (2019/20532-3).

\section{References}

1. Huang, C. et al. Clinical features of patients infected with 2019 novel coronavirus in Wuhan, China. The Lancet 395, 497-506, doi:10.1016/S01406736(20)30183-5 (2020).

2. Yang, Y. et al. Plasma IP-10 and MCP-3 levels are highly associated with disease severity and predict the progression of COVID-19. J Allergy Clin Immunol 146, 119-127.e114, doi:10.1016/j.jaci.2020.04.027 (2020).

3. Wang, Z., Yang, B., Li, Q., Wen, L. \& Zhang, R. Clinical Features of 69 Cases With Coronavirus Disease 2019 in Wuhan, China. Clin Infect Dis 71, 769777, doi:10.1093/cid/ciaa272 (2020).

4. Mehta, P. et al. COVID-19: consider cytokine storm syndromes and immunosuppression. Lancet 395, 1033-1034, doi:10.1016/S01406736(20)30628-0 (2020).

5. Ye, Q., Wang, B. \& Mao, J. The pathogenesis and treatment of the 'Cytokine Storm' in COVID-19. J Infect 80, 607-613, doi:10.1016/j.jinf.2020.03.037 (2020).

6. Ackermann, M. et al. Pulmonary Vascular Endothelialitis, Thrombosis, and Angiogenesis in Covid-19. N Engl J Med 383, 120-128, doi:10.1056/NEJMoa2015432 (2020).

7. Dolhnikoff, M. et al. Pathological evidence of pulmonary thrombotic phenomena in severe COVID-19. J Thromb Haemost 18, 1517-1519, doi:10.1111/jth.14844 (2020).

8. Varga, Z. et al. Endothelial cell infection and endotheliitis in COVID-19. Lancet 395, 1417-1418, doi:10.1016/S0140-6736(20)30937-5 (2020).

9. Marshall, J. C. Such stuff as dreams are made on: mediator-directed therapy in sepsis. Nat Rev Drug Discov 2, 391-405, doi:10.1038/nrd1084 (2003).

10. Angus, D. C. \& van der Poll, T. Severe sepsis and septic shock. N Engl J Med 369, 840-851, doi:10.1056/NEJMra1208623 (2013).

11. Salomao, R. et al. Bacterial sensing, cell signaling, and modulation of the immune response during sepsis. Shock (Augusta, Ga.) 38, 227-242, doi:10.1097/SHK.0b013e318262c4b0 (2012).

12. Iba, T. et al. The unique characteristics of COVID-19 coagulopathy. Crit Care 24, 360, doi:10.1186/s13054-020-03077-0 (2020).

13. Syed, F. et al. Excessive Matrix Metalloproteinase-1 and Hyperactivation of Endothelial Cells Occurred in COVID-19 Patients and Were Associated With the Severity of COVID-19. J Infect Dis 224, 60-69, doi:10.1093/infdis/jiab167 (2021).

14. Akalin, E. et al. Covid-19 and Kidney Transplantation. N Engl J Med 382, 2475-2477, doi:10.1056/NEJMc2011117 (2020).

15. Azzi, Y. et al. COVID-19 infection in kidney transplant recipients at the epicenter of pandemics. Kidney Internationa/98, 1559-1567, doi:10.1016/j.kint.2020.10.004 (2020).

16. Cravedi, P. et al. COVID-19 and kidney transplantation: Results from the TANGO International Transplant Consortium. Am J Transplant 20, 31403148, doi:10.1111/ajt.16185 (2020).

17. Requiao-Moura, L. R. et al. High mortality among kidney transplant recipients diagnosed with coronavirus disease 2019: Results from the Brazilian multicenter cohort study. PLoS One 16, e0254822, doi:10.1371/journal.pone.0254822 (2021).

18. Pecanha-Pietrobom, P. M. et al. The clinical course of hospitalized moderately ill COVID-19 patients is mirrored by routine hematologic tests and influenced by renal transplantation. PLoS One 16, e0258987, doi:10.1371/journal.pone.0258987 (2021).

19. Krieglstein, C. F. \& Granger, D. N. Adhesion molecules and their role in vascular disease. Am J Hypertens 14, 44s-54s, doi:10.1016/s08957061(01)02069-6 (2001).

20. AIRD, W. C. Spatial and temporal dynamics of the endothelium. Journal of Thrombosis and Haemostasis 3, 1392-1406, doi:https://doi.org/10.1111/j.1538-7836.2005.01328.x (2005).

21. Mazagova, M. et al. Growth differentiation factor 15 impairs aortic contractile and relaxing function through altered caveolar signaling of the endothelium. American Journal of Physiology-Heart and Circulatory Physiology 304, H709-H718, doi:10.1152/ajpheart.00543.2012 (2013). 
22. Wischhusen, J., Melero, I. \& Fridman, W. H. Growth/Differentiation Factor-15 (GDF-15): From Biomarker to Novel Targetable Immune Checkpoint. Front Immunol 11, doi:10.3389/fimmu.2020.00951 (2020).

23. Adela, R. \& Banerjee, S. K. GDF-15 as a Target and Biomarker for Diabetes and Cardiovascular Diseases: A Translational Prospective. J Diabetes Res 2015, 490842, doi:10.1155/2015/490842 (2015).

24. Feng, Y. et al. IL-9 Promotes the Development of Deep Venous Thrombosis by Facilitating Platelet Function. Thromb Haemost 118, 1885-1894, doi:10.1055/s-0038-1673614 (2018).

25. Leisman, D. E. et al. Cytokine elevation in severe and critical COVID-19: a rapid systematic review, meta-analysis, and comparison with other inflammatory syndromes. Lancet Respir Med 8, 1233-1244, doi:10.1016/S2213-2600(20)30404-5 (2020).

26. Goud, P. T., Bai, D. \& Abu-Soud, H. M. A Multiple-Hit Hypothesis Involving Reactive Oxygen Species and Myeloperoxidase Explains Clinical Deterioration and Fatality in COVID-19. Int J Biol Sci 17, 62-72, doi:10.7150/ijbs.51811 (2021).

27. Shi, H. et al. The inhibition of IL-2/IL-2R gives rise to CD8(+) T cell and lymphocyte decrease through JAK1-STAT5 in critical patients with COVID-19 pneumonia. Cell Death Dis 11, 429, doi:10.1038/s41419-020-2636-4 (2020).

28. Mancini, I. et al. The ADAMTS13-von Willebrand factor axis in COVID-19 patients. Journal of Thrombosis and Haemostasis 19, 513-521, doi:https://doi.org/10.1111/jth.15191 (2021).

29. Ebihara, T. et al. Adipocytokine Profile Reveals Resistin Forming a Prognostic-Related Cytokine Network in the Acute Phase of Sepsis. Shock 56, 718-726, doi:10.1097/SHK.0000000000001756 (2021).

30. Matsumoto, H. et al. The clinical importance of a cytokine network in the acute phase of sepsis. Sci Rep 8, 13995, doi:10.1038/s41598-018-32275-8 (2018).

31. Pierrakos, C. \& Vincent, J. L. Sepsis biomarkers: a review. Crit Care 14, R15, doi:10.1186/cc8872 (2010).

32. Terpstra, M. L., Aman, J., van Nieuw Amerongen, G. P. \& Groeneveld, A. B. Plasma biomarkers for acute respiratory distress syndrome: a systematic review and meta-analysis*. Crit Care Med 42,691-700, doi:10.1097/01.ccm.0000435669.60811.24 (2014).

33. Bandopadhyay, P. et al. Nature and Dimensions of Systemic Hyperinflammation and its Attenuation by Convalescent Plasma in Severe COVID-19. $J$ Infect Dis 224, 565-574, doi:10.1093/infdis/jiab010 (2021).

34. Liu, Y. et al. An inter-correlated cytokine network identified at the center of cytokine storm predicted COVID-19 prognosis. Cytokine 138, 155365, doi:10.1016/j.cyto.2020.155365 (2021).

35. Young, B. E. et al. Viral Dynamics and Immune Correlates of Coronavirus Disease 2019 (COVID-19) Severity. Clin Infect Dis 73, e2932-e2942, doi:10.1093/cid/ciaa1280 (2021).

36. Organization, W. H. Clinical management of COVID-19: interim guidance 2020).

37. Shannon, P. et al. Cytoscape: a software environment for integrated models of biomolecular interaction networks. Genome Res 13, 2498-2504, doi:10.1101/gr.1239303 (2003).

\section{Figures}



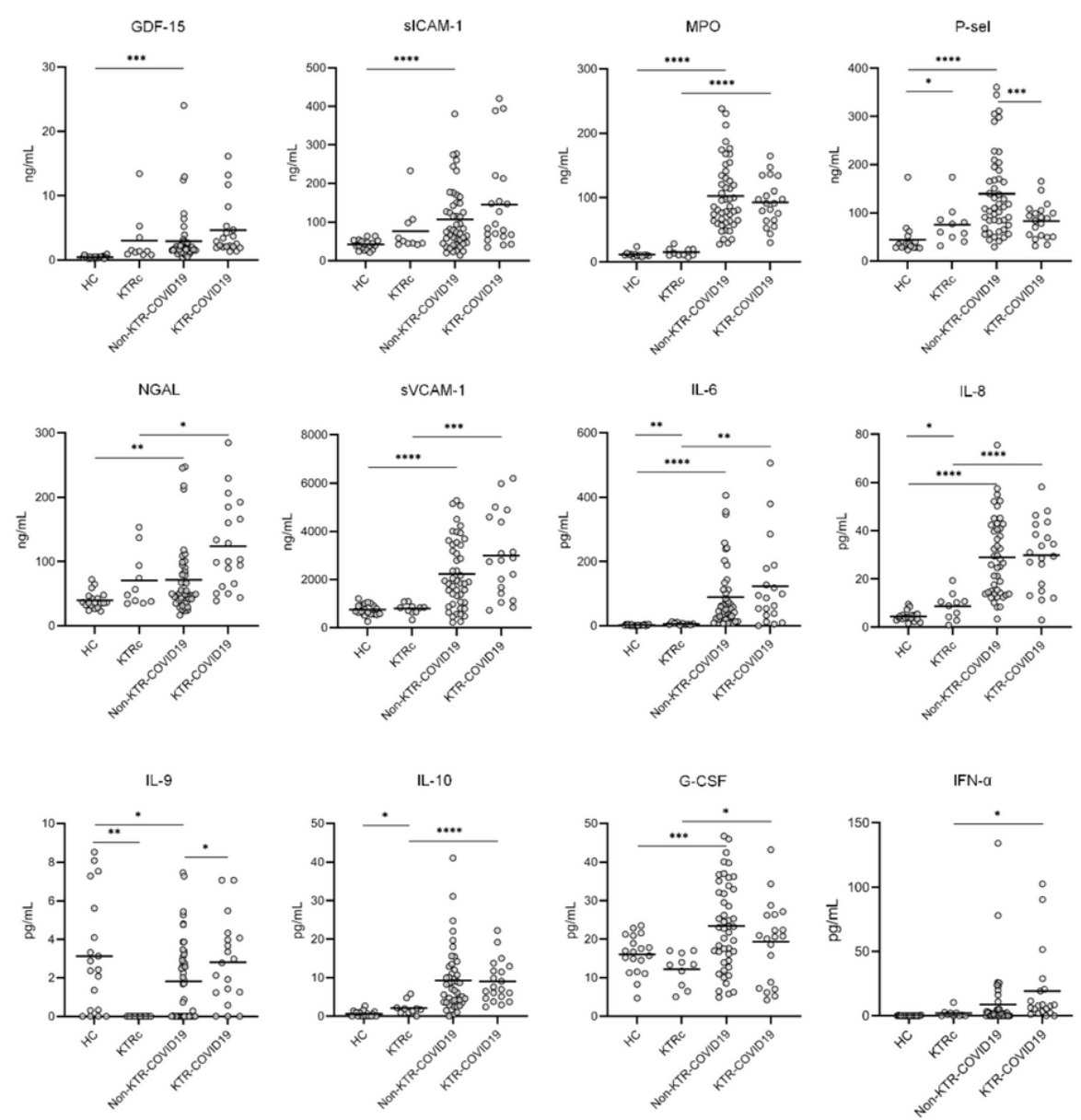

\section{Figure 1}

Comparison of the means of the different mediators in the groups of patients with COVID-19 and controls on the day of ward admission.

HC vs. KTRc: higher levels of P-Sel, SAA, IL-6, IL-8, and IL-10 and lower levels of ADAMTS-13, IL-2, and IL-9 in KTRs. HC vs. COVID-19 patients: higher levels of GDF-15, sICAM-1, MPO, P-Sel, NGAL, sVCAM-1, IL-6, IL-8, G-CSF, and IL-18 in COVID-19 patients. HC vs. non-KTR-COVID-19 patients: higher levels of GDF-15, sICAM-1, MPO, P-SEL, NGAL, sVCAM-1, IL-6, IL-8, G-CSF and lower levels of IL-9 in non-KTR-COVID-19 patients. KTRcs vs. KTR-COVID19 patients: higher levels of MPO, NGAL, sVCAM-1, IL-6, IL-8, IL-10, G-CSF, and IFN-a in KTR-COVID-19 patients. The levels of the mediators were similar between patients with COVID-19, regardless of KT status, except for significantly higher means of IL-9 and lower means of P-Sel in KTRs.

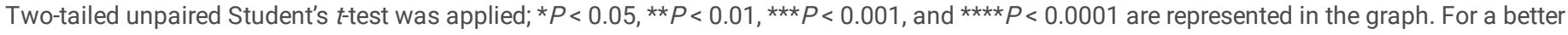
graphical representation, outliers were removed from NGAL, sVCAM-1, IL-6, IL-8, IL-9, IL-10, and IFN-a.

Abbreviations: ADAMTS-13, disintegrin, and metalloproteinase with thrombospondin motifs 13; G-CSF, granulocyte colony-stimulating factor; GDF-15, growth/differentiation factor; HC, healthy controls; IL, interleukin; KTRcs, kidney transplant recipients controls; KTR-COVID-19, kidney transplant recipients with COVID-19; MPO, myeloperoxidase; Myo, myoglobin; NGAL, neutrophil gelatinase-associated lipocalin; Non-KTR-COVID-19, non-kidney transplant recipients with COVID-19; P-Sel, P-selectin; SAA, serum amyloid A; sICAM-1, soluble intercellular adhesion molecule-1; sVCAM-1, soluble circulating vascular cell adhesion molecule-1; TNF-a, tumor necrosis factor-alpha. 
a

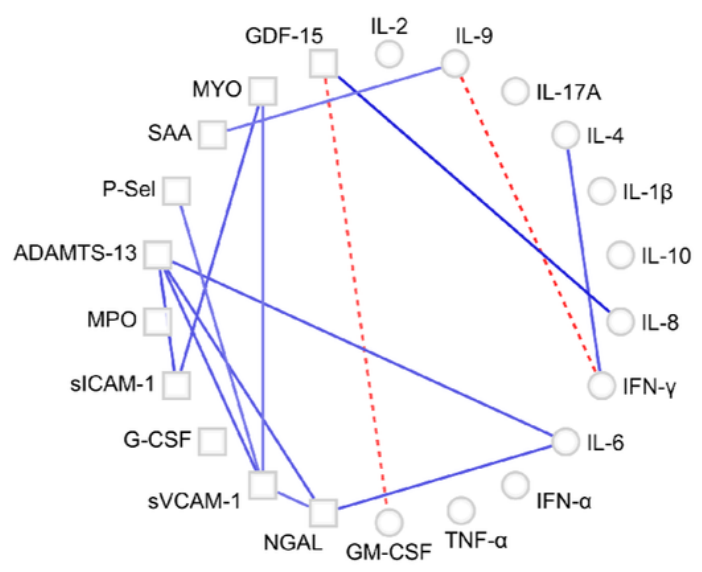

C
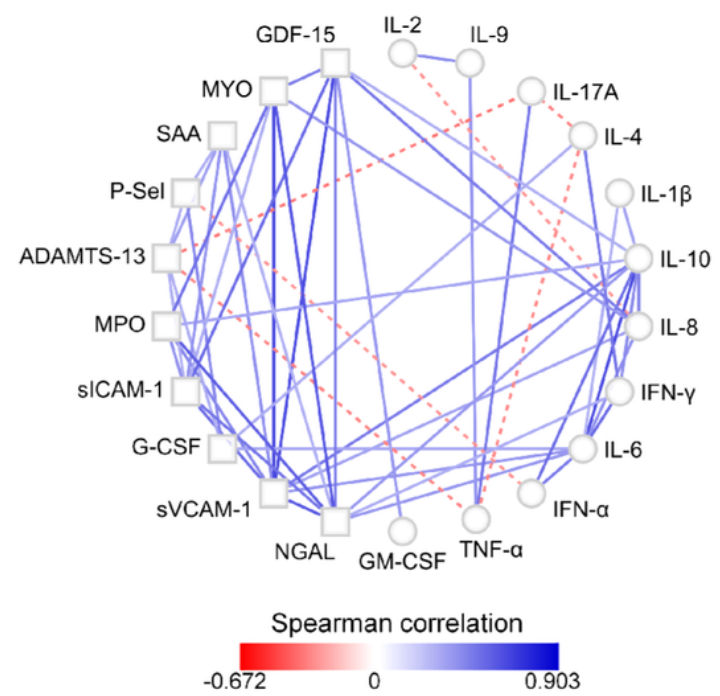

b

Kidney transplant recipients control

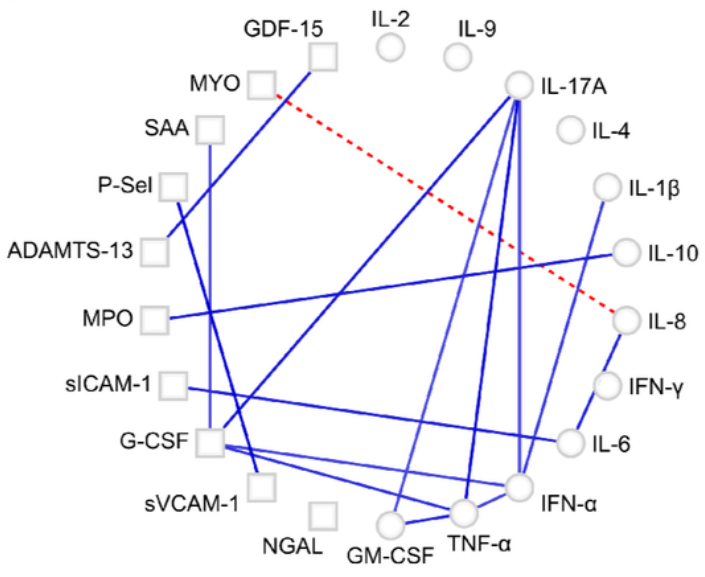

d $\quad$ KTR-COVID19 on first day

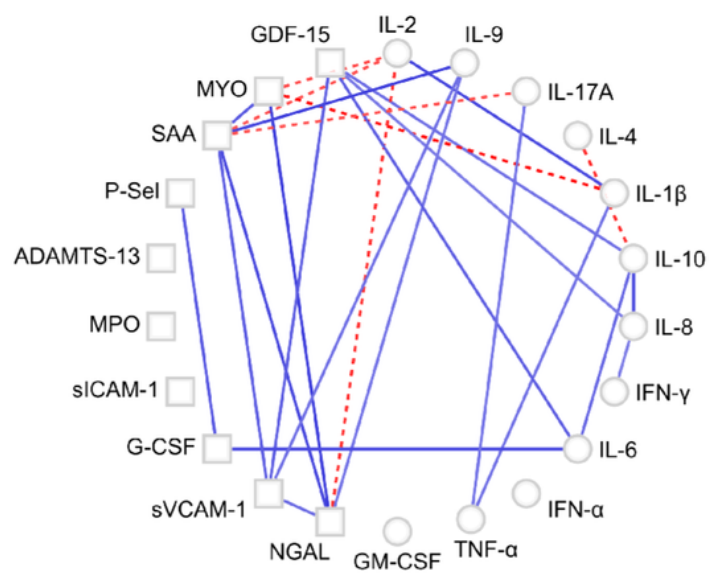

Figure 2

Correlation-based networks of cytokines and vascular mediators.

The two networks at the top of the figure show the results for healthy controls (a) and KTR controls (b). The other two networks show the results of the correlations between the mediators on the first day of hospital admission for non-KTR-COVID-19 (c) and KTR-COVID-19 patients (d). These networks allow visualization of the significant edges, which correspond to Spearman correlations with $r>|0.2|$ and $P<0.05$. The blue lines represent positive correlations, and dashed red lines represent negative correlations. The intensity of the edge colors indicates the strength of the correlations. The round nodes represent the cytokines and square vascular mediators. 

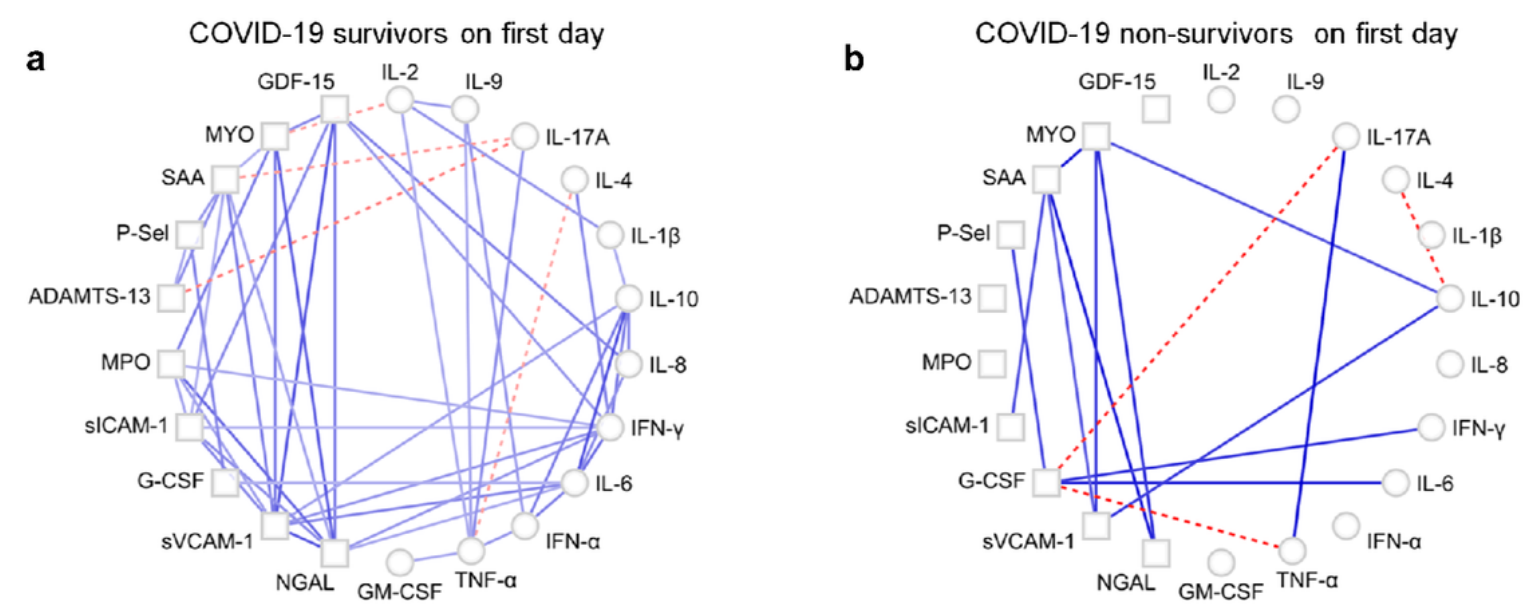

C COVID-19 survivors on the 3rd day

d COVID-19 non-survivors on the 3rd day
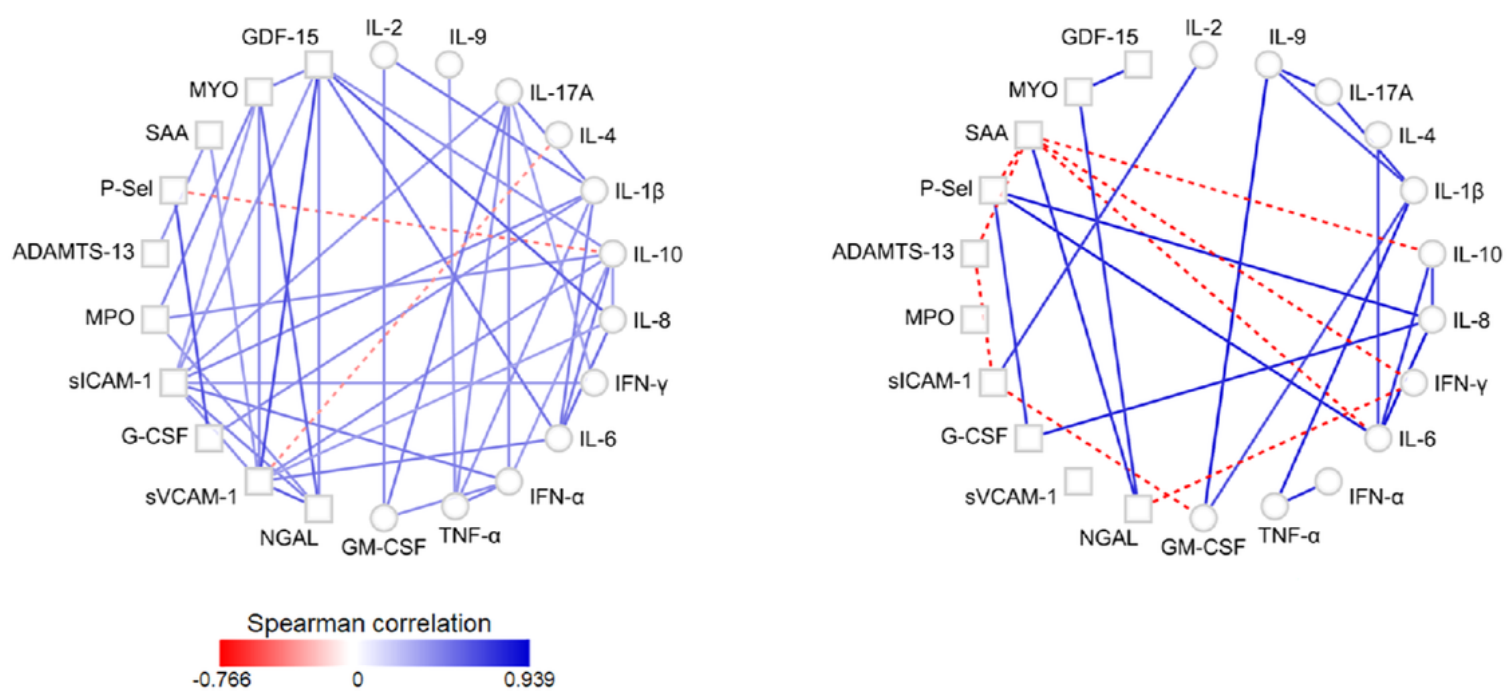

Figure 3

Correlation-based networks of cytokines and vascular mediators assessed on the first and third days in survivors and non-survivors.

The two networks at the top of the figure show the results based on the mediators on the first day in survivors (a) and non-survivors (b) with COVID-19. The other two networks show the results of the correlations between the mediators on the third day in survivors (c) and non-survivors (d) with COVID-19. These networks allow for visualization of the significant edges, which correspond to Spearman correlations with $r>|0.2|$ and $P<0.05$. The blue lines represent positive correlations, and dashed red lines represent negative correlations. The intensity of the edge colors indicates the strength of the correlations. The round nodes represent the cytokines and square vascular mediators.

\section{Supplementary Files}

This is a list of supplementary files associated with this preprint. Click to download.

- Supplementarymaterial1.pdf

- Supplementarymaterial2.pdf

- Supplementarymaterial3.pdf

- Supplementarymaterial4.pdf 